\title{
PARTIAL AORTECTOMY IN HYPOTHERMIC CIRCULATORY ARREST: METHOD OF CHOICE IN PATIENTS WITH AORTIC ARCH ATHEROMA
}

\author{
P. Lemke, MD, ${ }^{a}$ M. Schwab, MD, ${ }^{b}$ K. Hellberg, MD, ${ }^{a}$ C. Rieckmann, MD, ${ }^{c}$ H. Seibert, MD, ${ }^{a}$ and B. Urbanyi, MD, ${ }^{a}$ \\ Stuttgart and Ludwigsburg, Germany
}

Atherosclerotic lesions of the aorta, particularly in the region of the arch, are potential sources of cerebral and systemic embolization. ${ }^{1}$ Herein we describe 2 patients in whom successful surgical treatment was performed in deep hypothermic circulatory arrest by excision of the aortic arch atheroma and synthetic graft replacement.

Clinical summaries

PATIENT 1. A 39-year-old woman with pre-existing rheumatoid arthritis was admitted because of sudden-onset aphasia and a left brachiofacial hemiparesis. Long-term smoking and arterial hypertension were identified as cardiovascular risk factors. A hemostatic disorder could be excluded. According to the underlying disease of rheumatoid arthritis, elevated acute phase reactants (eg, C-reactive protein) and presence of rheumatoid factors (eg, immunoglobulin M rheumatoid factor) were found. Cranial computed tomography of the head at admission revealed three 1.2-cm large ischemic areas in the right basal layer, ganglion, and multiple small ischemic areas in the temporoparietal medullary layer. Further investigations, such as lumbar puncture and electrocardiography, showed no pathologic findings. The Doppler ultrasound examination of the internal carotid arteries and the vertebral arteries revealed an $80 \%$ and $60 \%$ stenosis of the left and the right vertebral arteries, respectively. Transesophageal echocardiography (TEE) showed an atherosclerotic plaque with superimposed mobile thrombotic components on the concavity of the posterior segment of the aortic arch (Fig 1). No further abnormalities of the aorta or of the cardiac chambers were found. At operation, the patient was placed on cardiopulmonary bypass with the use of arterial cannulation of the ascending aorta, which was free of atherosclerotic plaques according to information from the TEE. After the patient's body was cooled to a core temperature of $18^{\circ} \mathrm{C}$, her circulation was arrested for 38 minutes. The aortic

From the Department of Cardiovascular Surgery, Robert-BoschHospital, ${ }^{\mathrm{a}}$ the Dr Margarete Fischer-Bosch Institute of Clinical Pharmacology, ${ }^{\mathrm{b}}$ Stuttgart, and the Department of Internal Medicine II (Cardiology), General Hospital, ${ }^{\mathrm{C}}$ Ludwigsburg, Germany.

Received for publication July 15, 1999; accepted for publication Aug 3, 1999.

Address for reprints: Matthias Schwab, MD, Dr Margarete FischerBosch-Institute of Clinical Pharmacology, Robert-BoschKrankenhaus, Auerbachstrasse 112, D- 70376 Stuttgart, Germany (E-mail: matthias.schwab@ikp-stuttgart.de).

J Thorac Cardiovasc Surg 1999;118:1114-5

Copyright (C) 1999 by Mosby, Inc.

$0022-5223 / 99 \$ 8.00+0 \quad \mathbf{1 2 / 5 4 / 1 0 1 9 1 4}$

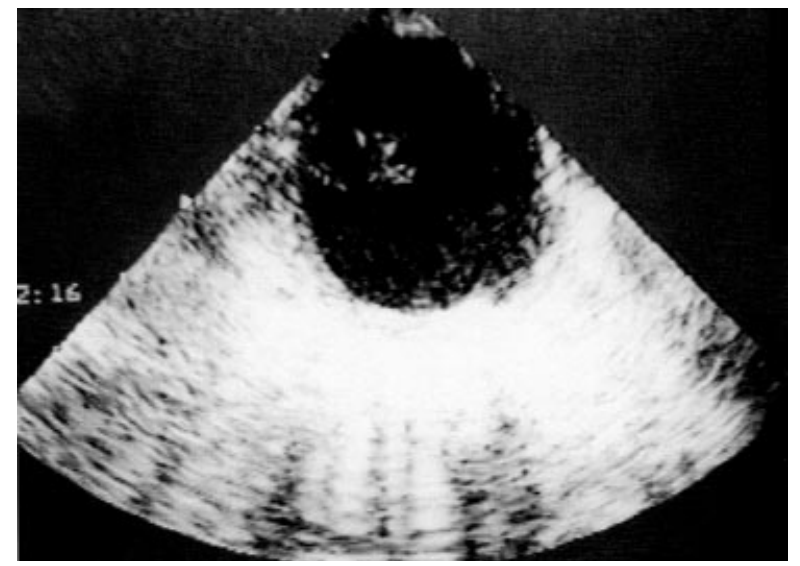

Fig 1. Preoperative transesophageal echocardiogram (transversal view) with protruding mobile thrombotic components superimposed on a broad-based focal atheroma on the concavity of the posterior segment of the aortic arch.

arch was incised from the innominate artery and extended to the small curvature of the aortic arch. Opposite the left common carotid artery, a focal atherosclerotic lesion $(6 \times 4 \mathrm{~cm}$ in diameter) was noted, which extended to the ligamentum Botalli with protruding atheroma components. The entire atheroma was excised. Reconstruction of the small curvature up to the descending aorta was performed with an impregnated Dacron patch (Fig 2). The postoperative course was uneventful. Histologic examination confirmed an atherosclerotic process with ulcerations. After discharge, a temporary anticoagulation was discontinued. Over a mid-term follow-up of 8 months, the patient has had no further neurologic events.

PATIENT 2. A 67-year-old woman with hypertension and diabetes had sudden-onset rotational vertigo with severe headache and emesis. The cranial computed tomogram revealed several new ischemic areas located in the cerebellum, probably caused by an embolic event. TEE showed an atherosclerotic plaque with mobile thrombotic material on the concavity of the aortic arch, opposite the ostia of the left carotid and subclavian arteries. No other causes of cerebral embolism were found. Doppler ultrasound examination revealed normal supra-aortic vessels. Surgical treatment consisted of careful arterial cannulation of the ascending aorta. Placement of the arterial cannula was based on information obtained from the TEE. The patient's body was cooled to a core temperature of $15.6^{\circ} \mathrm{C}$, and her circulation was arrested for 36 minutes. The aortic incision was begun ventral of the 
innominate artery and extended up to the descending aorta. One plaque ( $1 \mathrm{~cm}$ in diameter) without a thrombus was found on the concavity of the posterior segment of the aortic arch, and a further atherosclerotic plaque (approximately $5 \mathrm{~cm}$ in diameter) with thrombotic components was located opposite the ostia of the left carotid and subclavian arteries. The atherosclerotic lesions were excised, and the aortic arch was reconstructed with a Dacron patch. After an uneventful recovery, the patient had no evidence of further neurologic events at a follow-up time of 18 months. Histologic examination confirmed atherosclerotic lesions with ulcerative plaques.

Discussion. Atherosclerotic plaques of the aortic arch have been considered to be a rare cause of thromboembolic events and recurrent ischemic stroke. ${ }^{1}$ The prevalence of insertion of thrombi on the wall opposite the ostia of the aortic arch, also seen in our cases, is striking.

Optimal treatment strategies for atherosclerotic aortic plaques remain unclear. In some cases, anticoagulation with warfarin was considered to prevent thrombotic formations and, consequently, cerebral embolism. ${ }^{2}$ However, recurrent embolic events may occur despite anticoagulant therapy in patients who do not undergo surgical treatment of atheromas. ${ }^{3}$ On the other hand, in the literature, surgical treatment of symptomatic atherosclerotic lesions of the aortic arch was attempted in only a few cases. For example, Swanson and $\mathrm{Cohn}^{4}$ described an endarterectomy technique in deep hypothermic circulatory arrest as a safe procedure in a patient with 2 focal atheromas in the aortic arch. Laperche and colleagues $^{3}$ recommended that, despite its own risk, surgical removal of atherosclerotic plaques with thrombotic components does not appear to be associated with the high risk of surgical removal of aortic debris.

In accordance with our surgical procedure, Belden and colleagues $^{5}$ reported a successful aortectomy with synthetic graft replacement of the aortic arch in a patient with multiple cerebral embolism that had been caused by a broad-based, mobile, protruding atheroma in the transverse aortic arch.

In patients with atherosclerotic plaques of the aortic arch, (partial) aortectomy in deep hypothermic arrest therefore seems to be a safe approach and should be considered as the method of choice. To our knowledge, all described cases, including our patients, had an uneventful postoperative recovery and no further neurologic events.

For etiologic diagnosis of arterial embolism, several studies emphasize TEE as a reliable method to detect cardiac thrombi, patent foramen ovale (associated with paradoxic embolism), atrial septal aneurysm, or localized areas of aortic atherosclerosis. ${ }^{1,3}$ In our 2 patients, TEE permitted the precise localization of the atherosclerotic plaques and mobile thrombotic appositions.

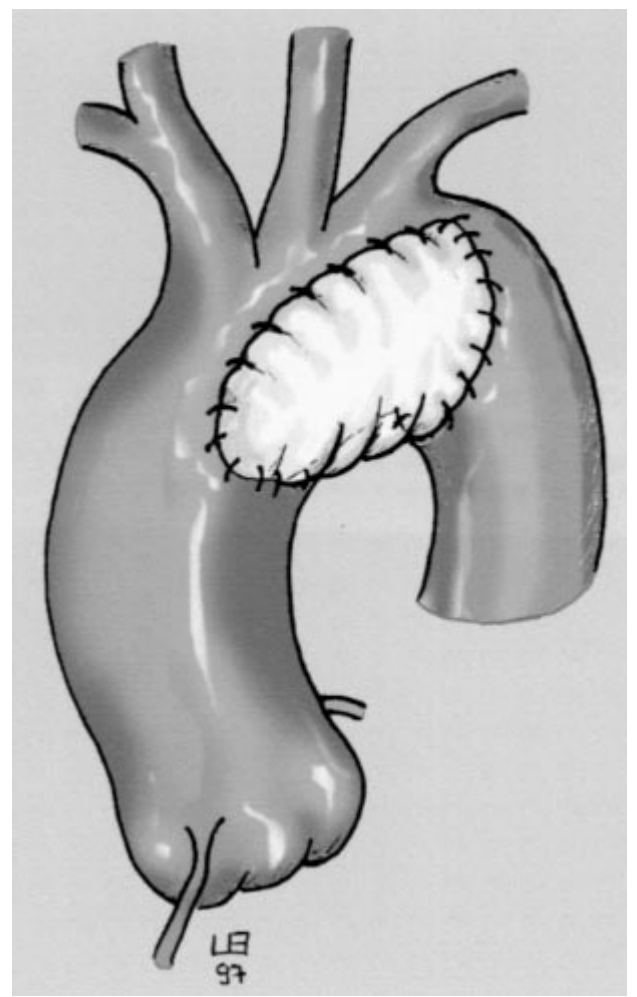

Fig 2. Artist's impression of the surgical procedure. Excision of the entire atheroma with subsequent reconstruction of the aortic wall with an impregnated Dacron patch.

\section{REFERENCES}

1. The French Study of Aortic Plaques in Stroke Group: atherosclerotic disease of the aortic arch as a risk factor for recurrent ischemic stroke. N Engl J Med 1996;334:1216-21.

2. Bansal RC, Pauls GL, Shankel SW. Blue digit syndrome: transesophageal echocardiographic identification of thoracic aortic plaque-related thrombi and successful outcome with warfarin. J Am Soc Echocardiogr 1993;6:319-23.

3. Laperche T, Laurian C, Roudaut R, Steg PG. Mobile thromboses of the aortic arch without aortic debris: a transesophageal echocardiographic finding associated with unexplained arterial embolism: the filiale echocardiographie de la Societé Francaise de Cardiologie. Circulation 1997;96:288-94.

4. Swanson SJ, Cohn LH. Excision of focal aortic arch atheroma using deep hypothermic circulatory arrest. Ann Thorac Surg 1995;60:457-8.

5. Belden JR, Caplan LR, Bojar RM, Payne DD, Blachmann P. Treatment of multiple cerebral emboli from an ulcerated, thrombogenic ascending aorta with aortectomy and graft replacement. Neurology 1997;49:621-2. 